\title{
SEQUENTIAL ESTIMATION OF THE SQUARE OF THE RAYLEIGH PARAMETER
}

\author{
Mohamed Tahir \\ Department of Mathematics, University of Sharjah, P.O. Box 27272, Sharjah, United Arab Emirates
}

Received 2014-02-11; Revised 2014-03-02; 2014-06-19

\section{ABSTRACT}

The problem addressed is that of sequentially estimating the square of the parameter of the Rayleigh distribution, subject to a weighted squared loss plus cost of sampling. We propose a sequential procedure and provide a second-order asymptotic expansion for the incurred regret. It is seen that the asymptotic regret is negative for a range of values of the parameter.

Keywords: Anscombe's Theorem, Excess Over the Stopping Boundary, Hölder's Inequality, Regret, Sequential Procedure

\section{INTRODUCTION}

Let $\mathrm{X}_{1}, \ldots, \mathrm{X}_{\mathrm{n}}$ denote independent observations to be taken sequentially up to a predetermined stage $n$ from the Rayleigh distribution with p.d.f:

$$
f_{\theta}(x)= \begin{cases}\frac{x}{\theta^{2}} \mathrm{e}^{-\frac{x^{2}}{2 \theta^{2}}} & \text { if } x>0 \\ 0 & \text { if not }\end{cases}
$$

where, $\theta$ is an unknown positive number. It is desired to estimate $\theta^{2}$, subject to the loss function considered by (Chow and Yu, 1981; Martinsek, 1988) that is Eqution 1:

$$
L_{a}\left(w_{n}, \theta^{2}\right)=a^{2} \theta^{4 \beta-4}\left[w_{n}-\theta^{2}\right]^{2}+n,
$$

where, a is a known positive number, determined by the cost of estimation relative to the cost of a single observation, $\beta>1$ is a given number and $\mathrm{w}_{\mathrm{n}}$ is an appropriate point estimate of $\theta^{2}$ (defined below). In practice, one might be interested in estimating the population variance. $\sigma^{2}=1 / 2(4-\pi) \theta^{2}$ or the population second moment $\mu_{2}=2 \theta^{2}$. Since both of these parameters are linear functions of $\theta^{2}$, it suffices to estimate $\theta^{2}$.

For observed values $x_{1}>0, \ldots, x_{\mathrm{n}}>0$, of $X_{1}, \ldots, X_{n}$, the log-likelihood function is:

$$
l_{n}(\theta)=\sum_{i=1}^{n} \ln x_{i}-2 n \ln \theta-\frac{1}{2 \theta^{2}} \sum_{i=1}^{n} \ln x_{i}^{2}
$$

For $\theta>0$. It follows that the maximum likelihood estimator of $\theta$ is:

$$
\hat{\theta}_{n}=\sqrt{\frac{1}{2 n} \sum_{i=1}^{n} X_{i}^{2}}=\sqrt{\bar{Y}_{n}}
$$

where, $\bar{Y}_{n}=\frac{1}{n} \sum_{i=1}^{n} Y_{i}$ with $Y_{i}=X_{\mathrm{i}}^{2} / 2, \mathrm{i}=1, \ldots, \mathrm{n}$ and where the random variables $Y_{1}, \ldots, Y_{n}$ are independent with common distribution the Exponential distribution with mean $\mu_{Y}=\theta^{2}$ and standard deviation $\sigma_{Y}=\theta^{2}$.

The risk incurred by estimating $\theta^{2}$ with $W_{n}=\hat{\theta}_{n}^{2}=\bar{Y}_{n}$ under the loss (1) is:

$$
R_{a}(n)=a^{2} \theta^{4 \beta-4} E\left[\left(\bar{Y}_{n}-\theta^{2}\right)^{2}\right]+n=\frac{a^{2} \theta^{4 \beta}}{n}+n
$$

For any fixed value of $a>0$, this risk is minimized with respect to $\mathrm{n}$ by choosing $\mathrm{n}$ as the greatest integer less than or equal to $n_{a}=a \theta^{2 \beta}=a \sigma_{Y}^{\beta}$; in which case, the minimum risk is Equation 2:

$$
R_{a}^{*}=R_{a}\left(n_{a}\right)=2 n_{a}=2 a \sigma_{Y}^{\beta}
$$


Since $\mathrm{n}_{\mathrm{a}}$ depends on the unknown value of $\theta$, there is no fixed-sample-size procedure that attains the minimum risk $R_{a}^{*}$ in practice. Therefore, we propose to use the sequential procedure $\left(T, \bar{Y}_{T}\right)$ which stops the sampling process after observing $Y_{1}, \ldots, Y_{T}$ and estimates $\theta^{2}$ by $W_{T}=\bar{Y}_{T}$, where Equation 3:

$T=\inf \left\{n \geq m_{a}: n>a\left(\frac{1}{n} \sum_{i=1}^{n}\left(Y_{i}-\bar{Y}_{n}\right)^{2}\right)^{\beta / 2}\right\}$

with $\mathrm{m}_{\mathrm{a}}$ being a positive integer. Note that the standard deviation based on $Y_{1}, \ldots, Y_{n}$ is used in (2) as the estimator of $\theta^{2}$, instead of $W_{n}=\bar{Y}_{n}$, since $\theta^{2}$ is also the standard deviation of $Y_{1}$.

If $\mathrm{m}_{\mathrm{a}}$ in (3) is such that $\delta \vee a \leq m_{a}=\mathrm{o}(a)$ as $a \rightarrow \infty$ for some $\delta>0$, then Equation 4 :

$$
E\left[\bar{Y}_{T}\right]=\mu_{Y}-\frac{\beta}{a} \sigma_{Y}^{1-\beta}+o\left(\frac{1}{a}\right)=\theta^{2}-\frac{\beta}{a \theta^{2 \beta-2}}+o\left(\frac{1}{a}\right)
$$

As a $\theta \theta$, by Martinsek (1988), since the skewness of $Y_{1}$ is equal to 2. This shows that $\bar{Y}_{T}$ is biased for large values of a. Thus, consider the biased-corrected estimator Equation 5:

$\theta_{n}^{*}=\bar{Y}_{n}+\frac{\beta}{a^{1 / \beta} n^{1-1 / \beta}}$

For $n \geq 1$, where $\beta>1$. The regret of the sequential procedure $\left(T, \theta_{T}^{*}\right)$ is defined as Equation 6:

$r_{a}\left(T, \theta_{T}^{*}\right)=E\left[L_{a}\left(T, \theta_{T}^{*}\right)\right]-R_{a}^{*}$

where, $R_{a}^{*}$ is as in (2). In this study we provide a secondorder asymptotic expansion, as as $a \rightarrow \infty$, for $r_{a}\left(T, \theta_{T}^{*}\right)$ and show that this regret is asymptotically negative if we choose $0<\theta<\sqrt[6]{(4 \beta-4) /(3.25 \beta+1)}$.

Starr and Woodroofe (1969) considered the case in which $\mathrm{X}_{1}, \mathrm{X}_{2}, \ldots$ are i.i.d. Normal random variables and showed that the regret of their procedure is $O(1)$. Then, Woodroofe (1977) showed that the regret is $0.5+\mathrm{o}(1)$ if $m_{a} \geq 4$. Martinsek (1983) extended Woodroofe's result to the nonparametric case. Tahir (1989) proposed a class of bias-reduction estimators of the mean of the oneparameter exponential family and provided an asymptotic second-order lower bound for the regret. Kim and Han (2009) considered estimation of the scale parameter of the Rayleigh distribution under general progressive censoring. Mousa et al. (2005; Prakash, 2013) focused on Bayesian prediction and Bayesian estimation for Rayleigh models.

\section{ASYMPTOTIC EXPANSION FOR THE REGRET OF THE SEQUENTIAL PROCEDURE}

Rewrite the stopping time $\mathrm{T}$ in (3) as Equation 7:

$t=\inf \left\{n \geq m_{a}: n\left(\frac{V_{n}}{n}\right)^{-1 / 2}>a\right\}$, where $V_{n}=\sum_{i=1}^{n}\left(Y_{i}-\bar{Y}_{n}\right)^{2}$

And let $U_{a}=t\left(V_{t} / t\right)^{-1 / 2}-a$ denote the excess over the stopping boundary. Chang and Hsiung (1979) showed that the excess $\mathrm{U}_{\mathrm{a}}$ converges in distribution to a random variable $\mathrm{U}$ as $\mathrm{a} \rightarrow \infty$.

\section{Lemma 1}

Let $\mathrm{T}$ be as in (3). Then $\frac{\mathrm{T}}{\mathrm{a}} \rightarrow \sigma_{\mathrm{Y}}^{\beta}=\theta^{2 \beta}$ w.p.1 as $\mathrm{a} \rightarrow$ $\infty$. Moreover:

$$
E[T]=a+v-1.375+o(1)
$$

As $a \rightarrow \infty$, where $v=E[U]$ is the asymptotic mean of the excess over the boundary.

\section{Proof}

The first assertion follows from Lemma 1 of Chow and Robbins (1985). For the second assertion:

$$
\begin{aligned}
E[T]= & \left.a+v-0.5-\frac{3}{8 \sigma_{Y}^{4}} E\left[\left(Y_{1}-\mu_{Y}\right)^{2}-\sigma_{Y}^{2}\right)^{2}\right]+o(1) \\
& =a+v-0.5-\frac{3}{8}(\kappa-1)+o(1) \\
& =a+v-1.375+o(1)
\end{aligned}
$$

As $a \rightarrow \infty$, by Chang and Hsiung (1979), using the fact that the kurtosis of $Y_{1}$ is $\kappa=\sigma_{Y}^{-4} E\left[\left(Y_{1}-\mu_{Y}\right)^{4}\right]=6$.

\section{Proposition 1}

Let $\theta_{n}^{*}$ be defined by (5) and let T be defined by (3) with ma being such that $\delta \vee a \leq m_{a}=$ o(a)as $a \rightarrow \infty$ for some $\delta>0$. Then, $E\left[\theta_{T}^{*}\right]=\theta^{2}+o(1 / a)$ as a $\rightarrow \infty$.

\section{Proof}

For $a>0$ Equation 8: 
$a E\left[\theta_{T}^{*}-\theta^{2}\right]=a E\left[\bar{Y}_{T}-\theta^{2}\right]+\beta E\left[\left(\frac{T}{a}\right)^{-(1-1 / \beta)}\right]$

The proposition follows by taking the limit as $a \rightarrow \infty$ in (8) and using (4) and the fact that $E\left[(T / a)^{-(l-1 / \beta)}\right] \rightarrow \sigma_{Y}^{I-\beta} \quad$ as $a \rightarrow \infty$ if $\beta>1$, by the first assertion of Lemma 1 and (2.2) of Martinsek (1983).

Let $r_{a}\left(T, \theta_{T}^{*}\right)$ be as in (6). Then Equation 9:

$$
\begin{aligned}
& r_{a}\left(T, \theta_{T}^{*}\right)=E\left[a^{2} \sigma_{Y}^{2 \beta-2}\left(\bar{Y}_{T}-\mu_{Y}\right)^{2}+T-2 a \sigma_{Y}^{\beta}\right] \\
& +2 \beta \sigma_{Y}^{2 \beta-2} a^{2-1 / \beta} E\left[\frac{1}{T^{1-1 / \beta}}\left(\bar{Y}_{T}-\mu_{Y}\right)\right] \\
& +\beta^{2} \sigma_{Y}^{2 \beta-2} E\left[\frac{a^{2-2 / \beta}}{T^{2-2 / \beta}}\right] \\
& =r_{a}\left(T, \bar{Y}_{T}\right)+2 \beta \sigma_{Y}^{2 \beta-2} E\left[\frac{a^{1-1 / \beta}}{T^{1-1 / \beta}} a\left(\bar{Y}_{T}-\mu_{Y}\right)\right] \\
& +\beta^{2} \sigma_{Y}^{2 \beta-2} E\left[\frac{a^{2-2 / \beta}}{T^{2-2 / \beta}}\right]
\end{aligned}
$$

\section{Lemma 2}

Let $\mathrm{T}$ be defined by (3) with $\mathrm{m}_{\mathrm{a}}$ being such that $\delta \vee a \leq m a=o(a)$ as $a \rightarrow \infty$ for some $\delta>0$ and with $\beta>1$. Then:

$$
E\left[\frac{a^{1-1 / \beta}}{T^{1-1 / \beta}} a\left(\bar{Y}_{T}-\mu_{Y}\right)\right]=\frac{2(1-\beta)}{\sigma_{Y}^{2 \beta+1}}-\frac{\beta}{\sigma_{Y}^{2 \beta-2}}+\mathrm{o}(1)
$$

As $\alpha \rightarrow \infty$.

\section{Proof}

First, observe that Equation 10:

$$
\begin{aligned}
& E\left[\frac{a^{1-1 / \beta}}{T^{1-1 / \beta}} a\left(\bar{Y}_{T}-\mu_{Y}\right)\right] \\
= & E\left[\left(\left(\frac{a}{T}\right)^{1-1 / \beta}-\frac{1}{\sigma_{Y}^{\beta-1}}\right) a\left(\bar{Y}_{T}-\mu_{Y}\right)\right]+\frac{1}{\sigma_{Y}^{\beta-1}} a E\left[\bar{Y}_{T}-\mu_{Y}\right]
\end{aligned}
$$

For $a>0$. Moreover Equation 11:

$$
a E\left[\bar{Y}_{T}-\mu_{Y}\right]=-\frac{\beta}{\sigma_{Y}^{\beta-1}}+o(1)
$$

As $a \rightarrow \infty$, by (4). Next, expand $g(y)=y^{1 / \tilde{\theta} 1}$ at $y=\sigma_{Y}^{\beta}$, substitute $y=T / a$ and multiply by $a\left(\vec{Y}_{T}-\mu_{Y}\right)$ to obtain Equation 12:

$$
\begin{aligned}
& \left(\frac{a^{1-1 / \beta}}{T^{1-1 / \beta}}-\frac{1}{\sigma_{Y}^{\beta-1}}\right)\left(\bar{Y}_{T}-\mu_{Y}\right) \\
& =\left(\frac{1}{\beta}-1\right) T_{*}^{1 / \beta-2}\left(\frac{T}{a}-\sigma_{Y}^{\beta}\right) a\left(\bar{Y}_{T}-\mu_{Y}\right)
\end{aligned}
$$

where, $\mathrm{T}_{*}$ is a random variable such that $\left|T . \sigma_{Y}^{\beta}\right| \leq\left|T / a-\sigma_{Y}^{\beta}\right|$. Next, rewrite $\mathrm{T}$ in (3) as $\mathrm{T}=\inf \{\mathrm{n} \geq$ $\mathrm{m}_{\mathrm{a}}: \mathrm{n}\left(\mathrm{V}_{\mathrm{n}} / \mathrm{n}\right)^{-\beta / 2}>\mathrm{a}$ \}, where, $\mathrm{V}_{\mathrm{n}}$ is as in (7) and let:

$$
U_{a}^{*}=T\left(\frac{V_{T}}{T}\right)^{-\beta / 2}-a
$$

Denote the excess over the stopping boundary. Expanding $\mathrm{h}(\mathrm{y})=\mathrm{y}^{-\beta / 2}$ at $y=\sigma_{Y}^{2}$, substituting $\mathrm{y}=\mathrm{V}_{\mathrm{T}} / \mathrm{T}$ and multiplying by $\mathrm{T}$ yields:

$$
\begin{aligned}
& T\left(\frac{V_{T}}{T}\right)^{-\beta / 2}=\frac{T}{\sigma_{Y}^{\beta}}-\frac{\beta}{2 \sigma_{Y}^{\beta+2}} \\
& \left(V_{T}-T \sigma_{Y}^{2}\right)+\frac{\beta(\beta+2)}{8 \lambda_{T}^{\beta / 2+2}} \frac{\left(V_{T}-T \sigma_{Y}^{2}\right)^{2}}{T}
\end{aligned}
$$

for $a>0$, where $\lambda_{T}$ is a random variable between $V_{T} / T$ and $\sigma_{Y}^{2}$. Furthermore, write:

$$
V_{T}=\sum_{i=1}^{T}\left(Y_{i}-\mu_{Y}\right)^{2}-T\left(\bar{Y}_{T}-\mu_{Y}\right)^{2}
$$

To obtain:

$$
\begin{aligned}
& U_{a}^{*}=\frac{T}{\sigma_{Y}^{\beta}}-a-\frac{\beta}{2 \sigma_{Y}^{\beta+2}}\left(W_{T}-T \sigma_{Y}^{2}\right) \\
& +\frac{\beta}{2 \sigma_{Y}^{\beta+2}} T\left(\bar{Y}_{T}-\mu_{Y}\right)^{2}+\frac{\beta(\beta+2)}{8 \lambda_{T}^{\beta / 2+2}} \frac{\left(V_{T}-T \sigma_{Y}^{2}\right)^{2}}{T}
\end{aligned}
$$

For a $>0$, where $W_{T}=\sum_{i=1}^{T}\left(Y_{i}-\mu_{Y}\right)^{2}$. It follows easily that Equation 13:

$$
\frac{T}{a}-\sigma_{Y}^{\beta}=\frac{\sigma_{Y}^{\beta}}{a}\left(U_{a}^{*}-\xi_{T}\right)+\frac{\beta}{2 a \sigma_{Y}^{2}}\left(W_{T}-T \sigma_{Y}^{2}\right)
$$

For $a>0$, where:

$$
\xi_{T}=\frac{\beta}{2 \sigma_{Y}^{\beta+2}} T\left(\bar{Y}_{T}-\mu_{Y}\right)^{2}+\frac{\beta(\beta+2)}{8 \lambda_{T}^{\beta / 2+2}} \frac{\left(V_{T}-T \sigma_{Y}^{2}\right)^{2}}{T}
$$

Substituting (13) in (12) yields Equation 14: 


$$
\begin{aligned}
& \left(\frac{a^{1-1 / \beta}}{T^{1-1 / \beta}}-\frac{1}{\sigma_{Y}^{\beta-1}}\right)\left(\bar{Y}_{T}-\mu_{Y}\right) \\
& =\left(\frac{1}{\beta}-1\right) \sigma_{Y}^{\beta} T_{*}^{1 / \beta-2}\left(U_{a}-\xi_{T}\right)\left(\bar{Y}_{T}-\mu_{Y}\right) \\
& +\left(\frac{1}{\beta}-1\right) \frac{\beta}{2 \sigma_{Y}^{2}} T_{*}^{1 / \beta-2}\left(W_{T}-T \sigma_{Y}^{2}\right)\left(\bar{Y}_{T}-\mu_{Y}\right) \\
& =\left(\frac{1}{\beta}-1\right) \sigma_{Y}^{\beta} I_{1}(a)+\frac{1-\beta}{2 \sigma_{Y}^{2}} I_{2}(a),
\end{aligned}
$$

Say. Let $S_{n}=Y_{1}+\ldots+Y_{n}, \mathrm{n} \geq 1$. Then Equation 15:

$$
\begin{aligned}
& E\left[\left|I_{1}(a)\right|\right]=E\left[\left|\frac{T_{*}^{1 / \beta-2}}{T}\left(U_{a}-\xi_{T}\right)\left(S_{T}-\mu_{Y} T\right)\right|\right] \\
& =\frac{\sigma_{Y}^{\beta}}{\sqrt{a \sigma_{Y}^{\beta}}} E\left[\mid\left(U_{a}-\xi_{T}\right) \frac{a}{T} T_{*}^{1 / \beta-2} \frac{\left(S_{T}-\mu_{Y} T\right)}{\left.\sqrt{a \sigma_{Y}^{\beta}} \mid\right]}\right. \\
& \leq \frac{\sqrt{\sigma_{Y}^{\beta}}}{\sqrt{a}} \sqrt{E\left[\left(U_{a}-\xi_{T}\right)^{2}\right]} \sqrt{E\left[T _ { * } ^ { 2 / \beta - 4 } ( \frac { a } { T } ) ^ { 2 } \left(\frac{S_{T}-\mu_{Y} T}{\left.\left.\sqrt{a \sigma_{Y}^{\beta}}\right)^{2}\right]}\right.\right.} \\
& \leq \frac{1}{\sqrt{a}} \sqrt{2 \sigma_{Y}^{\beta} E\left[U_{a}^{2}\right]+2 \sigma_{Y}^{\beta} E\left[\xi_{T}^{2}\right]} \\
& \sqrt{E\left[T _ { * } ^ { 2 / \beta - 4 } ( \frac { a } { T } ) ^ { 2 } \left(\frac{S_{T}-\mu_{Y} T}{\left.\left.\sqrt{a \sigma_{Y}^{\beta}}\right)^{2}\right]} \rightarrow 0\right.\right.} \rightarrow
\end{aligned}
$$

as $\mathrm{a} \rightarrow \infty$, by Hölder's inequality, the fact that $T_{*} \rightarrow \sigma_{Y}^{\beta}\left(\left|T_{*}-\sigma_{Y}^{\beta}\right| \leq\left|T / a-\sigma_{Y}^{\beta}\right| \rightarrow 0\right.$ w.p.1 since $\mathrm{T} / \mathrm{a} \rightarrow \sigma_{Y}^{\beta}, \quad \frac{S_{T}-\mu_{Y} T}{\sqrt{a \sigma_{Y}^{\beta}}}$ converges in distribution to a

Standard Normal random variable by Anscombe's theorem, the facts that $\mathrm{E}\left[\mathrm{U}_{\mathrm{a}}^{2}\right] \rightarrow \mathrm{E}\left[\mathrm{U}^{2}\right]<\infty$ and $E\left[\xi_{T}^{2}\right]=O(1) \quad a \rightarrow \infty$ and (2.3), (2.8) and (2.9) of Martinsek (1983). To evaluate $E\left[I_{2}(a)\right]$, observe that Equation 16:

$$
\begin{aligned}
& I_{2}(a)=\frac{2 a \sigma_{Y}^{\beta}}{T} T_{*}^{1 / \beta-2} \frac{\left(W_{T}-T \sigma_{Y}^{2}\right)\left(S_{T}-\mu_{Y} T\right)}{a \sigma_{Y}^{\beta}} \\
& =2 \sigma_{Y}^{\beta} \frac{a}{T} T_{*}^{1 / \beta-2}\left(\frac{W_{T}-\sigma_{Y}^{2} T}{\sqrt{a \sigma_{Y}^{\beta}}}+\frac{S_{T}-\mu_{Y} T}{\sqrt{a \sigma_{Y}^{\beta}}}\right)^{2} \\
& -2 \sigma_{Y}^{\beta} \frac{a}{T} T_{*}^{1 / \beta-2}\left(\frac{W_{T}-\sigma_{Y}^{2} T}{\sqrt{a \sigma_{Y}^{\beta}}}\right)^{2} \\
& -2 \sigma_{Y}^{\beta} \frac{a}{T} T_{*}^{1 / \beta-2}\left(\frac{S_{T}-\mu_{Y} T}{\sqrt{a \sigma_{Y}^{\beta}}}\right)^{2} \\
& \stackrel{\text { in distribution }}{\longrightarrow} 2 \sigma_{Y}^{1-2 \beta}(2 Z)^{2} \\
& -2 \sigma_{Y}^{1-2 \beta} Z^{2}-2 \sigma_{Y}^{1-2 \beta} Z^{2}=4 \sigma_{Y}^{1-2 \beta} Z^{2}
\end{aligned}
$$

As $a \rightarrow \infty$, by Anscombe's theorem and the fact that $\mathrm{T}_{*}{ }^{\circledR} \sigma_{\mathrm{Y}}^{\beta} \quad$ w.p. 1 as $\mathrm{a} \rightarrow \infty$ where $\mathrm{Z}$ is a random variable having the Standard Normal distribution. Thus Equation 17:

$$
\mathrm{E}\left[\mathrm{I}_{2}(\mathrm{a})\right]=4 \sigma_{\mathrm{Y}}^{1-2 \beta}+\mathrm{o}(1)
$$

As $a \rightarrow \infty$, by (16) and (2.3) and (2.4) of Martinsek (1983). Taking expectation in (14) and using (15) and (17) yields Equation 18:

$$
\mathrm{E}\left[\left(\frac{\mathrm{a}^{1-1 / \beta}}{\mathrm{T}^{1-1 / \beta}}-\frac{1}{\sigma_{\mathrm{Y}}^{\beta-1}}\right) \mathrm{a}\left(\overline{\mathrm{Y}}_{\mathrm{T}}-\mu_{\mathrm{Y}}\right)\right]=\frac{2(1-\beta)}{\sigma_{\mathrm{Y}}^{2 \beta+1}}+\mathrm{o}(1)
$$

$a \rightarrow \infty$. The lemma follows by taking the limit, as $a \rightarrow \infty$, in (10) and using (11) and (18).

Theorem 1. Let $\mathrm{T}$ be defined by (3) with $\mathrm{m}_{\mathrm{a}}$ being such that $\delta \vee a \leq m_{a}=\mathrm{o}(a)$ as $a \rightarrow \infty$ for some $\delta>0$ and $\beta>1$. Let the regret of the biased-corrected procedure $\left(T, \theta_{T}^{*}\right)$ be as in (6). Then:

$$
r_{a}\left(T, \theta_{T}^{*}\right)=3.25 \beta^{2}+\beta-\frac{4 \beta(\beta-1)}{\theta^{6}}+o(1)
$$

As $\alpha \theta \theta$.

\section{Proof}

First Equation 19:

$$
\begin{aligned}
& \mathrm{r}_{\mathrm{a}}\left(\mathrm{T}, \overline{\mathrm{Y}}_{\mathrm{T}}\right)=\mathrm{E}\left[\mathrm{a}^{2} \sigma_{\mathrm{Y}}^{2 \beta-2}\left(\overline{\mathrm{Y}}_{\mathrm{T}}-\mu_{\mathrm{Y}}\right)^{2}+\mathrm{T}\right] \\
& -2 \mathrm{a} \sigma_{\mathrm{Y}}^{\beta}=5.25 \beta^{2}+\beta+\mathrm{o}(1)
\end{aligned}
$$

As $a \rightarrow \infty$ if $\delta>1$, by Martinsek (1988). Next, take the limit, as a $\rightarrow \infty$, in (9) and use (19), Lemma 2 and the fact that:

$$
E\left[\frac{a^{2-2 / \beta}}{T^{2-2 / \beta}}\right]=\frac{1}{\sigma_{Y}^{2 \beta-2}}+o(1)
$$

as $a \rightarrow \infty$ if $\delta>1$, by the first assertion of Lemma 1 and (2.2) of Martinsek (1983), to complete the proof.

\section{NEGATIVE ASYMPTOTIC REGRET}

Theorem 1 shows that the biased-corrected procedure $\left(T, \mu_{T}^{*}\right)$ has a lower asymptotic regret than the procedure $\left(T, \bar{Y}_{\mathrm{T}}\right)$. Also, the asymptotic regret of the procedure $\left(T, \mu_{T}^{*}\right)$ is negative if Equation 20:

$0<\theta<\sqrt[6]{\frac{4 \beta-4}{3.25 \beta+1}} \equiv \theta_{\beta}$ 
Table 1. Asymptotic regret for various choices of $\beta>1$ and $0<\theta<\theta_{\beta}$ (see (20))

\begin{tabular}{|c|c|c|c|}
\hline $\mathrm{b}$ & $\mathrm{q}_{\mathrm{b}}$ & $\mathrm{q}$ & Asymptotic regret \\
\hline 1.5 & 0.836 & 0.2 & -46866.1880000 \\
\hline 1.5 & 0.836 & 0.3 & -4106.4138000 \\
\hline 1.5 & 0.836 & 0.4 & -723.6093800 \\
\hline 1.5 & 0.836 & 0.5 & -183.1875000 \\
\hline 1.5 & 0.836 & 0.7 & -16.6870790 \\
\hline 2.0 & 0.901 & 0.2 & -124985.0000000 \\
\hline 2.0 & 0.901 & 0.3 & -10958.9370000 \\
\hline 2.0 & 0.901 & 0.4 & -1938.1250000 \\
\hline 2.0 & 0.901 & 0.5 & -497.0000000 \\
\hline 2.0 & 0.901 & 0.8 & -15.5175780 \\
\hline 2.0 & 0.901 & 0.9 & -0.0534114 \\
\hline 5.0 & 0.988 & 0.3 & -109653.12 .00000 \\
\hline 5.0 & 0.988 & 0.4 & -19445.0000000 \\
\hline 5.0 & 0.988 & 0.6 & -1628.4276000 \\
\hline 5.0 & 0.988 & 0.7 & -593.7387800 \\
\hline 5.0 & 0.988 & 0.9 & -64.2841140 \\
\hline 10 & 1.012 & 0.2 & -5624665.0000000 \\
\hline 10 & 1.012 & 0.4 & -87555.6250000 \\
\hline 10 & 1.012 & 0.7 & -2724.9495000 \\
\hline 10 & 1.012 & 0.8 & -1038.2910000 \\
\hline 10 & 1.012 & 0.9 & -342.4035100 \\
\hline 10 & 1.012 & 1.0 & -25.0000000 \\
\hline 15 & 1.020 & 0.2 & -13124254.0000000 \\
\hline 15 & 1.020 & 0.3 & -1151517.1000000 \\
\hline 15 & 1.020 & 0.5 & -53013.7500000 \\
\hline 15 & 1.020 & 0.7 & -6393.6322000 \\
\hline 15 & 1.020 & 0.9 & -834.3582000 \\
\hline 15 & 1.020 & 1.0 & -93.7500000 \\
\hline
\end{tabular}

This means that for the values of $\theta$ in the interval $(0$, $\left.\theta_{\beta}\right)$ with $\beta>1$, the sequential procedure $\left(T, \mu_{T}^{*}\right)$ performs better, for large values of a, than the best fixed-samplesize procedure $\left(n_{a}^{*}, \bar{Y}_{n_{a}^{*}}\right)$, where $\mathrm{n}_{\mathrm{a}}^{*}$ is the greatest integer less than or equal to $n_{a}=a \theta^{2 \beta}$ (see Table 1).

\section{CONCLUSION}

We have proposed a sequential procedure for estimating the square of the shape parameter of the Rayleigh distribution and provided a second-order asymptotic expansion for the incurred regret. It is seen that the proposed procedure performs better than the best fixed-sample-size procedure if the shape parameter lies in a specific subinterval of the positive real numbers.

For future research, it would be worth considering Bayesian sequential estimation of a function of the shape parameter of the Rayleigh distribution, in which the focus will be on finding a sequential procedure and approximating the Bayes regret, as well as comparing the proposed procedure with existing procedures.

\section{REFERENCES}

Chang, I.S. and C.A. Hsiung, 1979. Approximations to the expected sample size of certain sequential procedures. Proceedings of the Conference on, Recent Developments in Statistical Methods and Applications, (CRDS' 79), Taipei, Institute of Mathamatic Academi Sinica, pp: 71-82.

Chow, Y.S. and H. Robbins, 1985. On the asymptotic theory of fixed-width sequential confidence intervals for the mean. Annal. Math. Stat., 36: 457-462. DOI: 10.1007/978-1-4612-5110-1_19

Chow, Y.S. and K.F. Yu, 1981. The performance of a sequential procedure for the estimation of the mean. Annal. Stat., 9: 189-198.

Kim, C. and K. Han, 2009. Estimation of the scale parameter of the Rayleigh distribution under general progressive censoring. J. Korean Stat. Society, 38: 239-246. DOI: 10.1016/j.jkss.2008.10.005

Martinsek, A.T., 1983. Second order approximation to the risk of a sequential procedure. Annal. Stat., 11: 827-836. 
Martinsek, A.T., 1988. Negative regret, optional stopping and the elimination of outliers. J. Am. Stat. Assoc., 83: 160-163.

Mousa, M.A. M. Ali and S.A. Al-Sagheer, 2005. Bayesian prediction for progressively Type-II censored data from Rayleigh model. Commun. Stat. Theory Meth., 34: 2353-2361. DOI: $10.1080 / 03610920500313767$

Prakash, G., 2013. Bayes estimation in the inverse Rayleigh model. Electron. J. App. Stat. Anal., 6: 6783. DOI: $10.1285 /$ i20705948v6n1p67.
Starr, N. and M. Woodroofe, 1969. Remarks on sequential point estimation. Proc. Nat. Acad. Sci. USA., 63: 285-288.

Tahir, M., 1989. An asymptotic lower bound for the local minimax regret in sequential point estimation. Anal. Stat., 17: 1335-1346.

Woodroofe, M., 1977. Second order approximations for sequential point and interval estimation. Anal. Stat., 5: 984-995. 